Relations industrielles

Industrial Relations

\title{
GOMPERS, édité par Gérard Emmanuel Stern, New Jersey, Prentice-Hall Inc., Englewood Cliffs, 1971, 178 pp.
}

\section{André Roy}

Volume 26, numéro 3, 1971

URI : https://id.erudit.org/iderudit/028263ar

DOI : https://doi.org/10.7202/028263ar

Aller au sommaire du numéro

Éditeur(s)

Département des relations industrielles de l'Université Laval

ISSN

0034-379X (imprimé)

1703-8138 (numérique)

Découvrir la revue

Citer ce compte rendu

Roy, A. (1971). Compte rendu de [GOMPERS, édité par Gérard Emmanuel Stern, New Jersey, Prentice-Hall Inc., Englewood Cliffs, 1971, 178 pp.] Relations industrielles / Industrial Relations, 26(3), 784-786.

https://doi.org/10.7202/028263ar

Tous droits réservés @ Département des relations industrielles de l'Université Laval, 1971
Ce document est protégé par la loi sur le droit d'auteur. L'utilisation des services d'Érudit (y compris la reproduction) est assujettie à sa politique d'utilisation que vous pouvez consulter en ligne.

https://apropos.erudit.org/fr/usagers/politique-dutilisation/ 
quel ont évolué les relations de pouvoir entre une commission scolaire, un syndicat et une association d'enseignants d'une ville du New Jersey.

Il s'agit essentiellement d'une recherche sociologique dans laquelle l'Auteur identifie les modes de comportement caractéristiques de chacune des parties en présence au fur et à mesure que les relations de pouvoir entre elles sont modifiées.

Le cas de cette ville du New Jersey est typique de l'évolution générale des relations du travail dans le secteur public au cours des dix dernières années aux Etats-Unis. Au début de la période considérée, la balance du pouvoir penche nettement du côté de la partie patronale alors qu'il n'existe aucune législation régissant les relations du travail dans le secteur public. Les relations entre les parties sont alors caractérisées par la prise de décision unilatérale de l'employeur dans tous les domaines et les seuls avantages obtenus par les enseignants le sont grâce au paternalisme généreux de l'employeur. Lors de cette période initiale les enseignants sont groupés non pas en un syndicat mais dans une association plus ou moins dominée par l'employeur.

Par la suite, au fur et à mesure que les enseignants prennent conscience de leur situation, ils deviennent plus militants et commencent à s'organiser en un syndicat authentique. Les rivalités entre le syndicat et l'association divisent le corps professoral mais les enseignants gagnent néanmoins plus de pouvoirs et l'équilibre des forces commence à changer.

La résistance de la commission scolaire à accepter la détermination conjointe des conditions de travail de ses enseignants au moyen des négociations collectives associée à l'absence de législation du travail, entraîna les enseignants dans un militantisme encore plus considérable et l'inévitable arriva: les enseignants firent une grève de 11 jours au terme de laquelle la commission scolaire eut à signer malgré elle une convention collective. Le différentiel de la relation de pouvoir était maintenant disparu et la situation devint ce qu'elle est dans la plupart des cas lorsque les employés des secteurs privés ou publics décident de se syndiquer et de revendiquer la détermination conjointe de leurs conditions de travail. De plus, comme c'est également le cas général, une élection entre le syndicat et l'association permit d'écarter définitivement cette dernière du champ des relations du travail.

L'objectif visé par l'Auteur, consciemment ou non, est de démontrer que l'absence d'une législation compréhensive autorisant la négociation collective et prévoyant des mécanismes de représentation des employés ainsi que de résolution des conflits est un prérequis nécessaire et indispensable pour que les relations du travail se développent de façon satisfaisante pour toutes les parties impliquées. Ce livre, quoique publié en 1970, a été écrit antérieurement au passage de la législation adoptée par le New Jersey en 1969 et qui prévoit actuellement de tels mécanismes. La question du droit de grève n'y est cependant pas traitée et l'intérêt, pour un Canadien du moins, en est d'autant diminué, puisque ce livre traite finalement de questions qui ont été résolues depuis longtemps dans la majeure partie des législations provinciales.

Jean BOIVIN

GOMPERS, édité par Gérald Emmanuel Stern, New Jersey, Prentice-Hall Inc., Englewood Cliffs, 1971, 178 pp.

Parmi les pionniers les plus connus du syndicalisme américain, il faut placer Samuel Gompers, fondateur de la Fédération américaine du travail dont il demeura le président jusqu'à sa mort en 1924.

On pense le connaître, parce qu'on en a beaucoup entendu parler, qu'on s'y réfère encore de temps en temps. Est-ce si vrai? La lecture de l'ouvrage, qui s'intitule simplement Gompers, est de nature à faire voir combien, au fond, la connaissance qu'on peut avoir de l'homme et du chef syndical est passablement superficielle.

$\mathrm{Au}$ départ, toutefois, est-il à propos de signaler cette collection publiée par Gérald Emmanuel Stearn, sous la rubrique générale 《The Great Lives》 qui réunit en un seul volume une autobiographie tirée des écrits de l'homme même, l'opinion controversée de ses contemporains et le jugement de l'histoire.

D'extraction juive, Gompers était né dans les slums de Londres à la demie du XIXe siècle. En 1863, il émigre avec sa 
famille à New York. Déjà, avant de quitter la Grande-Bretagne, il avait connu le travail en fabrique, s'initiant au métier de cigarier dont il fut toujours fier. Beaucoup plus tard, se rappelant ses jeunes années, il écrivait: «La vie en fabrique compte parmi mes souvenirs les plus agréables. Je vivais vraiment dans la joie. Je trouvais une satisfaction aristocratique dans le travail ».

Aujourd'hui, on est enclin à considérer Gompers comme un syndicaliste réactionnaire, comme l'homme qui a lié indissolublement le syndicalisme américain au système capitaliste. Et pourtant, comme la plupart des premiers leaders ouvriers du XIXe siècle, il y est venu par l'étude des doctrines marxiste et socialiste, par la pratique du radicalisme, à preuve sa participation, au cours de la décennie 1870-1880, au mouvement en faveur de la journée de huit heures, son intervention un peu plus tard, en faveur des anarchistes de l'émeute du marché au foin de Chicago auprès du gouverneur de l'Etat des Illinois qui dévoilent un aspect bien particulier de son caractère : son extrême sensibilité.

La pensée syndicale de Gompers s'est forgée peu à peu au gré des événements, des luttes qu'il a eu à subir en tant que président de la centrale syndicale, de la situation dans laquelle vivait la masse des travailleurs américains, de l'opposition que manifestaient les employeurs et l'opinion publique en général contre le mouvement syndical naissant, toutes choses qui l'ont amené à prendre partie pour le syndicalisme de métier, à dénoncer le mouvement populiste qui cherchait à attirer les masses laborieuses dans l'action politique, à combattre tour à tour le socialisme, le bolchévisme, à manifester, chose curieuse, d'évidentes sympathies pour le fascisme italien de Benito Mussolini, à s'opposer à l'impérialisme, mais en s'appuyant sur des arguments qui ne pouvaient que conduire aux formes modernes et renouvelées du colonialisme économique actuel.

Son attitude envers les Noirs qu'il méprisait souverainement est bien caractéristique de sa mentalité. Parce que les employeurs les utilisaient comme briseurs de grève, Gompers les menaçait de ses foudres, leur promettant à l'occasion une « haine raciale pire que celles qu'ils n'avaient connues ». De même, la persistance pendant toute sa carrière de syndicaliste de sa lutte contre la politique d'immigration du gouvernement américain.

Gompers avait les pieds fixés au sol. Il connaissait bien l'art du possible. C'est cela sans doute qui explique que, une fois engagé dans l'action syndicale quotidienne, il ait rejeté toute idéologie et combattu le socialisme. Il n'aimait guère les intellectuels et les révolutionnaires pantouflards et ceux-ci le lui rendaient bien.

Sa conception de l'action syndicale était simple: obtenir toujours davantage pour les travailleurs. On lui posa, un jour qu'il témoignait devant un comité du Congrès, la question suivante : «La lutte des syndicats prendra-t-elle fin quand l'ouvrier aura touché la rétribution complète de son travail ? 》. La réponse de Gompers ne se fit pas attendre : «Elle ne cessera jamais ... Les travailleurs ne s'arrêteront jamais dans leurs efforts pour obtenir une vie meilleure pour euxmêmes, pour leurs épouses, pour leurs enfants, pour l'humanité. Il y aura toujours lutte quotidienne pour obtenir une vie meilleure 》. Au cours du même témoignage, il traduisit sa pensée d'une façon encore plus concrète: «Je ne dis pas $\$ 4.00$ ou $\$ 8.00$ par jour ou un montant quelconque de dollars par jour ou 8 heures ou 7 heures par jour ou un nombre d'heures quelconque par jour, mais la fin, c'est d'obtenir les meilleures conditions possibles pour les travailleurs ». Dans sa simplicité, pareille réponse était profondément humaine.

Autrement dit, Gompers croyait à une espèce de progrès illimité, mais à un progrès accompli étape par étape et il croyait aussi que le syndicalisme était l'instrument essentiel pour pouvoir $y$ participer dans une mesure valable. «Les travailleurs américains, disait-il, se rendent compte des conditions dans lesquelles ils vivent. Ils comprennent les grandes concentrations de l'industrie et les coalitions financières. Ils se sont rendu compte que, individuellement, ils n'ont aucune chance de défendre leurs droits, de faire redresser leurs griefs ou d'améliorer leurs conditions de vie ».

Comme il avait été mêlé de près aux premières tentatives des travailleurs américains pour unir leurs efforts, Gompers savait que le syndicalisme n'aurait pas la partie facile, qu'il avait comme adversaires, non seulement la presque totalité des employeurs et l'ensemble de la bourgeoisie, mais tout autant les agriculteurs 
et souvent les travailleurs eux-mêmes. Il savait aussi que, avec ses trois millions de membres, le syndicalisme américain n'était pas tellement représentatif, qu'il ne pesait pas lourd dans la balance. C'est pourquoi, sans doute par nécessité d'user sans cesse de tactiques et de stratagèmes, il fut fondamentalement opportuniste, qu'il rejeta vite aux oubliettes ses idées socialisantes et son radicalisme du début. On peut même se demander si son attachement vraiment démesuré au syndicalisme de métier ne peut pas s'expliquer par la crainte de voir le mouvement syndical courir trop tôt aux aventures politiques comme il l'était alors sans cesse sollicité par les groupes socialistes et les tenants des théories populistes. Peut-être estimait-il aussi que la syndicalisation des ouvriers spécialisés et des manœuvres de la grande industrie en voie d'expansion rapide s'avérait impossible à son jugement dans l'état des esprits et de la législation de l'époque.

$C$ 'est pourquoi, devenu le chef incontesté de la grande centrale syndicale, sans renoncer à une certaine forme d'un radicalisme verbal bien proche de la démagogie, il se montra dans la pratique un homme modéré singulièrement habile dans l'art de la retraite.

D'ailleurs, et le jugement de ses contemporains le confirme bien, Gompers a dû être un homme fort tiraillé. Des quatre points cardinaux, il ne pouvait trouver appui que d'un seul côté. A l'époque où il dirigeait la Fédération américaine du travail, il est bien certain que les employeurs et les hommes politiques ne le prisaient pas et qu'ils ne recoururent à ses services qu'à l'occasion des situations d'urgence, comme la guerre mondiale, par exemple, qu'il fallait bien faire accepter au peuple. Pour ce qui était alors la gauche américaine, c'était le grand adversaire, le traître. Quant à la masse ouvrière elle-même, elle était indifférente, apathique, à preuve cette déclaration de l'épouse d'un briseur de grève que le syndicat des peintres de Chicago avait condamné à l'amende et fait expulser de ses rangs en 1901: «M. Gompers! Je lui accorde qu'il est un administrateur habile. S'il travaillait dans son métier de cigarier, il gagnerait $\$ 15.00$ par semaine; il ne toucherait pas $\$ 1,500$. par année. Il n'irait pas se reposer les méninges à Cuba. Sa cave n'est pas vide. Ses enfants ne sont pas forcés de rester à la maison parce qu'ils n'ont pas de vêtements à se mettre sur le dos pour aller à l'école ». Par ailleurs, bon tribun, il soulevait les foules qui parfois l'adulaient. Fort mal pourvu au physique, il faisait un sujet magnifique pour les caricaturistes de son temps. Il avait de la présence d'esprit. On raconte que, au cours d'une entrevue avec le président T. Roosevelt, dans le feu de la discussion, celui-ci lui dit à brûle-pourpoint en frappant du poing son pupitre : "Sachez, M. Gompers, que je suis le président des Etats-Unis ». Imitant le geste de Roosevelt, Gompers répondit du tac au tac: «Sachez, M. le Président, que je suis le président de la Fédération américaine du travail».

Si le mouvement ouvrier a conservé jusqu'ici la marque profonde de la longue présidence de son fondateur, la réputation historique de Gompers n'est guère bonne, mais, pour les quatre Auteurs qui sont appelés à la barre, John R. Commons, Philip S. Foner, Daniel Bell et Benjamin Stoldberg, il fut, malgré ses défauts et ses limites, à la hauteur de la situation qui lui a été faite par les événements. Pendant 40 ans, la Fédération américaine du travail, ce fut la figure bien typique de Gompers. Il savourait les victoires de ses syndicats affiliés, souffrait de leurs échecs et excellait dans l'art de les défendre envers et contre tous.

Benjamin Stoldberg le compare à Moïse. Comme le héros de la Bible, par le syndicalisme, il a tiré le monde ouvrier américain de l'esclavage pour le conduire dans le désert et lorsque celui-ci fut en vue de la terre promise, Gompers s'en est allé, sans avoir contemplé sans doute du haut de la montagne l'avenir qui s'annonçait.

En attendant sur cet homme, dont le souvenir s'efface de la mémoire des générations nouvelles, le jugement définitif de l'histoire, peut-on dire avec le même auteur que «sa mort a révélé qu'il n'était pas un prophète, que sa vision des choses était limitée par son tempérament querelleur et que, pour le même motif, on ne peut le considérer comme un penseur. Il fut un chef de groupe à la personnalité forte, dont la droiture d'esprit un peu étroite ne fut jamais troublée par les doutes philosophiques et les rêves utopiques $\gg$. 